\title{
Hybride Wissensarbeit
}

\author{
Sarah Oeste-Reiß1 · Eva Bittner ${ }^{2}$ |zabel Cvetkovic ${ }^{2}$ Andreas Günther ${ }^{1}$ Jan Marco Leimeister ${ }^{1,3}$. \\ Lucas Memmert ${ }^{2} \cdot$ Anja Ott $^{1} \cdot$ Bernhard Sick $^{4} \cdot$ Kathrin Wolter ${ }^{1}$
}

Angenommen: 7. April 2021 / Online publiziert: 30. April 2021

(C) Der/die Autor(en) 2021

\section{Zusammenfassung}

Aufgrund der Fortschritte im Bereich der künstlichen Intelligenz (KI) ergeben sich neue Gestaltungsmöglichkeiten zur Reorganisation von Wissensarbeit an der Schnittstelle von Menschen und Maschine. Durch die Verschmelzung von menschlicher und künstlicher Intelligenz können komplementäre Stärken zum Lösen von Arbeitsaufgaben gebündelt werden. Neuartige Wissensarbeitssysteme sind erforderlich, die Wissensarbeitende sowohl bei der Ausübung von Routineaufgaben als auch Nichtroutineaufgaben unterstützen.

Der Schlagwortartikel umreißt die Grundlagen von Wissensarbeit und arbeitet die Charakteristika von Routineaufgaben und Nichtroutineaufgaben innerhalb von Arbeitsprozessen auf. Es werden Grenzen klassischer IT-gestützter Wissensarbeitssysteme als Werkzeuge umrissen, die Wissensarbeitende im Arbeitsprozess unterstützen. Aufbauend darauf diskutiert der Artikel, dass technologische Fortschritte eine arbeitsprozessintegrierte und personalisierte Unterstützung von Wissensarbeitenden erlauben. Dazu verweist der Artikel auf die Charakteristika von kollaborativ interaktiv lernenden Systemen. Darauf aufbauend werden Hybride Wissensarbeitssysteme vorgestellt, die sowohl das KI-unterstützte menschliche Lernen als auch das Mensch-unterstützte maschinelle Lernen unterstützen. In diesem Zusammenhang beschreibt der Artikel definitorische Grundlagen von menschlicher, künstlicher und hybrider Intelligenz, stellt drei Archetypen von Mensch-Maschine-Aufgaben innerhalb von hybriden Wissensarbeitssystemen vor und umreißt drei Gestaltungsdimensionen solcher Systeme. Mittels eines Praxisbeispiels zum kollaborativen Schreiben von Journalist*innen beschreibt der Artikel exemplarisch die hybride Wissensarbeit. Der Artikel schließt mit einem Ausblick auf zukünftige Forschungsbedarfe.

\section{Von IT-gestützten Wissensarbeitssystemen hin zu Hybriden Wissensarbeitssystemen}

Wissensarbeit bezieht sich auf den Wissensanteil einer Arbeitsaufgabe. Dies umfasst das benötigte Domänenwissen, den Arbeitsprozess und -aufgabe sowie die Aktivitäten zum Ausführen der Aufgabe [4]. Wissensarbeit ist besonders, da

Sarah Oeste-Reiß

oeste-reiss@uni-kassel.de

1 Fachgebiet Wirtschaftsinformatik, Universität Kassel, Kassel, Deutschland

2 Arbeitsgruppe Wirtschaftsinformatik, Sozio-Technische Systemgestaltung, Universität Hamburg, Hamburg, Deutschland

3 Institut für Wirtschaftsinformatik, Universität St. Gallen, St. Gallen, Schweiz

4 Intelligent Embedded Systems Group, Universität Kassel, Kassel, Deutschland sie sowohl Routine- als auch Nichtroutineaufgaben umfasst [11].

- Routineaufgaben sind wiederholend, automatisierbar und programmierbar. Sie gründen auf explizierbarem deklarativen Faktenwissen (,know what“) [12]. Der Wissenstransfer und -erwerb kann digital oder papierbasiert erfolgen - u. a. über Daten und Dokumentationen in Form von Büchern, Bedienungsanleitungen oder FAQ [13]. Beispiele finden sich in der Wiedergabe und $\mathrm{Zu}$ sammenführung von Faktenwissen (z. B. Rechnungserstellung, Terminvereinbarung).

- Nichtroutineaufgaben sind vielfältig und umfassen Einzelfallentscheidungen und -lösungen. Sie gründen auf schwer explizierbarem Erfahrungswissen (,know how“) [12]. Der Wissenstransfer und -erwerb erfolgt über Lernund Anwendungsprozesse. Diese sind gekennzeichnet durch eine soziale und häufig informelle Interaktion mit anderen Wissensträgern und der Umwelt, bei der u.a. erfahrungsbasiert Faktenwissen vernetzt und angewendet wird [2]. Beispiele finden sich in der Anwendung, 
Analyse, Beurteilung und Schaffung von Wissen (z.B. Mitarbeitereinarbeitung, Geschäftsmodellentwicklung, Mentoring).

Aus organisationaler Perspektive wird durch die Ausführung von Arbeitsaufgaben Wissen entwickelt, gesichert und zwischen Wissensarbeitenden transferiert. Dazu benötigen Wissensarbeitende Fähigkeiten, die sie zum lösungsorientierten Handeln, zu Kollaboration mit anderen Wissensträgern und zum lebenslangen Lernen befähigen [15].

Werkzeuge, die Wissensarbeitende hierbei unterstützen sind Wissensmanagementsysteme. Solche Systeme dienen der Identifikation, dem Erwerb, der Entwicklung, der Speicherung, der Pflege, der Nutzung und dem Transfer von Wissen [2]. Sie integrieren die Verwaltung sowohl von explizitem als auch von implizitem Wissen [5], das zur Lösung von Routine- und Nichtroutineaufgaben herangezogen wird. Als Strategien zum Teilen von Wissen kann zwischen der Kodifizierungs- und die Personalisierungsstrategie unterschieden werden. Erstere zielt auf das Sammeln, Speichern und Bereitstellen von explizitem Wissen mittels Datenbanken, Dokumentenmanagement oder Intranet [5, 7]. Letztere zielt auf die Befähigung von Wissensarbeitenden, ihr implizites Erfahrungswissen IT-gestützt über Wissensnetzwerke und Groupware-Systeme zu kommunizieren [7]. Solche Systeme dienen oft als Nachschlagewerke, in denen Wissensarbeitende proaktiv Wissen zur Lösung von Arbeitsaufgaben suchen und die Wissensbestände einspeisen und aktuell halten müssen.

Heutzutage erlauben Technologien, dass intelligente Systeme zunehmend arbeitsprozessintegriert und personalisiert Wissen bereitstellen und aktuell halten können. Voraussetzung hierfür ist ein lebenslanges Lernen des Systems, das auf einem „Human-in-the-loop“-Modell gründet.
Solche Systeme werden zunehmend in der Lage sein, mit unvollständigen Beispieldaten beim Lern- und Optimierungsprozess zur Entwicklungszeit umzugehen. Kollaborativ interaktiv lernende Systeme, sogenannte CIL-Systeme, gründen auf diesem Ansatz und verfügen über lebenslange Lernfähigkeiten [14]. Technische Systeme, die auf CILTechniken beruhen sind:

- lernend: d.h. selbstorganisierte Verbesserung der eigenen Wissensbasis und zugehöriger Lernprozesse [14];

- kollaborativ: d.h. wechselseitige auf ein gemeinsames Ziel ausgerichtete Einbindung intelligenter Systeme und Menschen in den Lernprozess [14];

- interaktiv: d.h. Informations- und Wissensaustausch erfolgt in beide Richtungen [14].

Technische Systeme, die auf CIL-Techniken basieren bieten das Potenzial, Wissensarbeitenden aktuelle Wissensbestände und personalisierte Wissensabrufe zu ermöglichen. Durch ihr selbstorganisiertes Lernen liefern sie Potenziale, um in implizite Wissenstransformationsprozesse vorzustoßen, welche bisher überwiegend zwischen Menschen möglich waren [2, 14]. Im Vergleich zu Wissensmanagementsystemen benötigen sie weniger dedizierte Wissensaufbereitung durch menschliche Systemadministratoren und versprechen durch ihre Kontextadaptivität Wissensarbeitenden personalisierte Wissensrepräsentation bereitzustellen. Weiterhin liefern sie Potenziale für eine arbeitsprozessintegrierte Nutzung, da sie mit menschlichen Nutzern kollaborieren können (z. B. Verifizierung von Beispieldaten, Übergabe/-nahme von Arbeitsständen).

Deshalb benötigen wir eine neue Generation von Wissensarbeitssystemen, die sowohl ein lebenslanges Lernen des technischen Systems als auch ein lebenslanges Lernen des Wissensarbeitenden (soziales System) gewährleisten.
Abb. 1 Hybrides Wissensarbeitssystem (HWA-System)
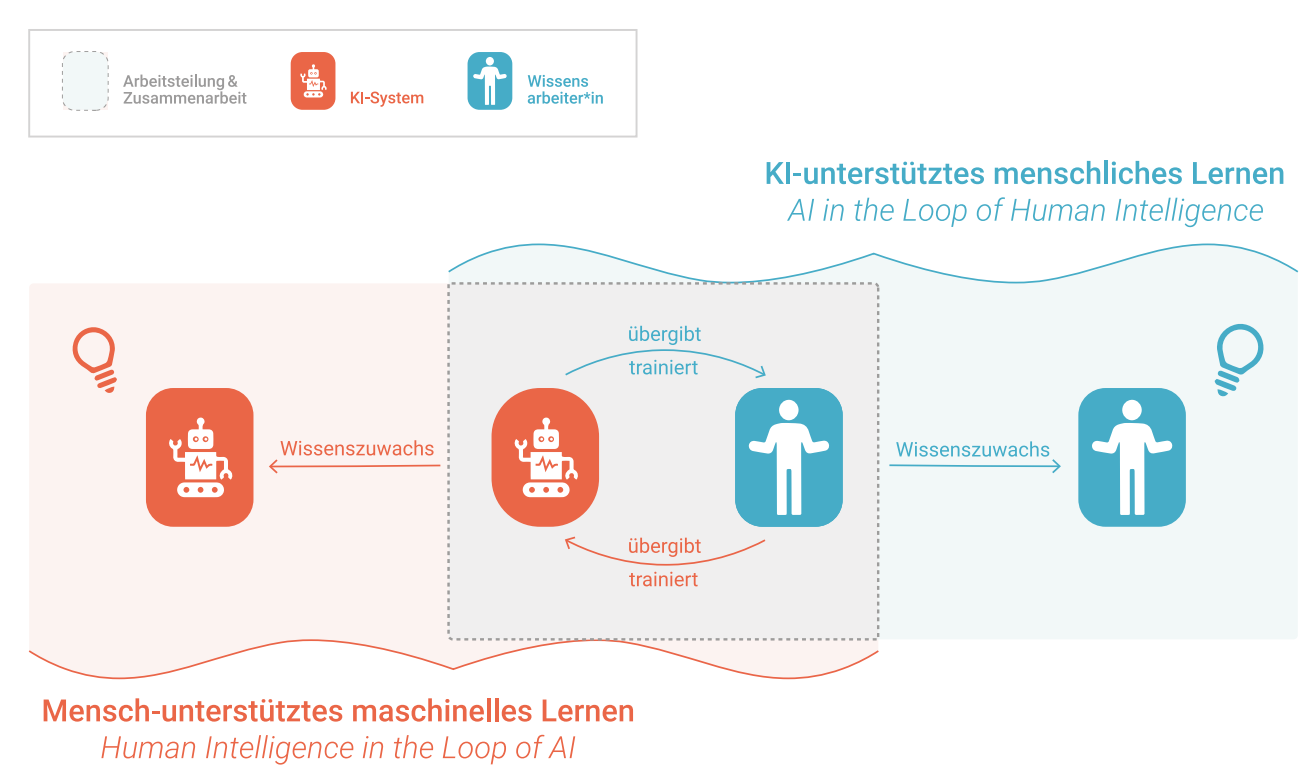
Diese Systeme bezeichnen wir im Folgenden als Hybride Wissensarbeitssysteme (HWA-Systeme). HWA-Systeme ermöglichen:

- KI-unterstütztes menschliches Lernen (,AI in the Loop of Human Intelligence"): Das technische KI-System nutzt CIL-Techniken. Es fungiert arbeitsprozessintegriert als Lerncoach und trainiert Wissensarbeitende mit dem Ziel einer personalisierten Wissensvermittlung zur Lösung von Arbeitsaufgaben. Es erfolgt eine Automatisierung und Augmentierung von Routine- und Nichtroutineaufgabenbestandteilen.

- Menschunterstütztes maschinelles Lernen („Human Intelligence in the Loop of AI"): Der Mensch fungiert arbeitsprozessintegriert als Lerncoach und trainiert das technische KI-System mit dem Ziel einer Verifizierung von Wissen.

Hieraus ergibt sich ein wiederkehrender Kreislauf von Übergaben und Training zwischen Wissensarbeitenden und KI-System und folglich ein neues Verständnis von Zusammenarbeit und Arbeitsteilung (siehe Abb. 1). Es bedarf einer menschzentrierten Gestaltung der Lern- und Zusammenarbeitsprozesse.

\section{Merkmale und Gestaltungsdimensionen Hybrider Wissensarbeitssysteme}

Die Grundidee von HWA-Systemen ist es, mittels Hybrider Intelligenz Wissensarbeitende fortwährend zum Erwerb und Transfer von Wissen zur Ausübung ihrer Arbeitsaufgaben zu befähigen. Wissensarbeitende sollen kognitiv im Arbeitsprozess bei der Ausübung von Arbeitsaufgaben entlastet und unterstützt werden. Hierzu werden komplementärere Stärken von Wissensarbeitenden und KI-System genutzt, sodass menschliche und künstliche Intelligenz verschmelzen.

Dies bedingt eine Verschiebung der Arbeitsteilung von Wissensarbeitenden und KI-System und vergrößert den Anteil der Aufgaben, die von Mensch und KI-System im Team erledigt werden können. HWA-Systeme bilden die Plattform für eine gesellschaftlich-wünschenswerte Neuverteilung von Arbeit, die durch KI-basierte Automatisierung und Augmentierung von Aufgabenbestandteilen gekennzeichnet ist. Ein Schlüsselelement, das dies ermöglicht ist das lebenslange Lernen sowohl von Wissensarbeitenden als auch vom KI-System. Lebenslanges Lernen wird durch die Gestaltung hybrider Zusammenarbeitspraktiken ermöglicht, die in HWA-Systemen implementiert sind. Zur Gestaltung sind einerseits Kenntnisse über die Fähigkeiten von Wissensarbeitenden und KI-System und andererseits die Analyse und Zergliederung des Arbeitsprozesses und insbesondere der Aufgabenbestandteile erforderlich.
Eine Definition von Intelligenz gibt Aufschluss über die Fähigkeiten und Stärken der jeweiligen Wissensträger.

- Menschliche Intelligenz bezieht sich auf die Fähigkeit zu lernen, zu verstehen und sich mithilfe des eigenen Wissens an die Umgebung anzupassen [9]. Sie weist Stärken im Bereich des Erfahrungswissens und bei der Bewältigung von Nichtroutineaufgaben auf.

- Künstliche Intelligenz bezieht sich auf die Fähigkeit eines Systems, externe Daten korrekt zu interpretieren, von ihnen zu lernen und das Gelernte zu nutzen und um bestimmte Ziele und Aufgaben durch flexible Anpassung zu erreichen [8]. Sie weist Stärken im Bereich des Faktenwissens und bei der Bewältigung von Routineaufgaben auf.

Hybride Zusammenarbeitspraktiken nutzen sogenannte Hybride Intelligenz, bei der menschliche und künstliche Intelligenz verschmelzen. Diese verspricht durch geeignete Kombination der menschlichen und künstlichen Intelligenzen ein Team zu schaffen, welches die individuellen, komplementären Stärken bündelt und Schwächen kompensiert. Somit können gemeinsame Ziele erreicht und Probleme gelöst oder besser gelöst werden, als dies dem Menschen oder der KI eigenständig möglich wäre [1, 6]. Der Ansatz verfolgt eine gegenseitige Ergänzung (Augmentierung) und nicht Ersetzung von Wissensarbeitenden durch die KI [1]. Im Fokus stehen dynamische Umgebungen und Probleme, deren Lösung domänenspezifisches Wissen bedürfen und die keine spezifische Grundwahrheit (,ground truth“) besitzen und somit bisher typischerweise dem menschlichen Intellekt vorbehalten waren $[1,6]$.

Da Arbeitsprozesse und -aufgaben von Wissensarbeitenden sowohl aus Routine- als auch Nichtroutineaufgaben bestehen, zielen HWA-Systeme auf hybride Zusammenarbeitspraktiken mit folgenden Archetypen von Aufgaben [16]:

- Automatisierung: Routineaufgabenbestandteile werden identifiziert, für die vollständige Beispieldaten für Lernund Optimierungsprozesses des KI-Systems existieren. Diese Aufgaben werden so automatisiert, dass ein Deskilling von Wissensarbeitenden vermieden wird.

- Verifizierung: Aufgabenbestandteile werden identifiziert, für die keine oder nur unvollständige Beispieldaten existieren. Wissensarbeitende verifizieren dieses Wissen.

- Augmentierung: Routine- und Nichtroutineaufgabenbestandteile werden identifiziert, die aus Gründen mangelnder Akzeptabilität gegenüber gesellschaftlichen Werten und Normen nicht automatisiert werden sollen. Mittels Augmentierung werden Wissensarbeitende beispielsweise in Kreativitätsprozessen unterstützt und entlastet.

Zur Gestaltung hybrider Zusammenarbeitspraktiken, die Hybride Intelligenz erschließen und nutzen, ist eine soziotechnische Gestaltungsperspektive notwendig. HWA-Syste- 
me sind Arbeitssysteme [3, 10], die als eine Art Marktplatz für eine Allokation von Arbeitsaufgaben und -ständen fungieren. Gestaltungsdimensionen sind:

- das soziale Subsystem (Wissensarbeitende);

- das technische Subsystem (CIL-Techniken) sowie

- Geschäftsprozesse (hybride Zusammenarbeitspraktiken).

In Letzterem erfolgt die eigentliche Arbeitsteilung und Zusammenarbeit zwischen Wissensarbeitenden und KISystem. Gestaltungsmethoden des sogenannten Collaboration Engineering [10, 17], einem Ansatz zur Entwicklung und zur Umsetzung von Kollaborationsprozessen, die von Nichtkollaborationsexperten ausgeführt werden können, um hochwertige, wiederkehrende Aufgaben zu erfüllen, liefern Werkzeuge um einen Arbeitsprozess hinsichtlich (i) des zu erbringenden Arbeitsergebnisses zu analysieren; (ii) in Aufgabenbestandteile zu zergliedern; (iii) Arbeitsstände und Übergaben zu definieren; (iv) Zusammenarbeitsaktivitäten zu definieren. Folglich sind HWA-Systeme soziotechnische Systeme, die Zusammenarbeits- und Lernpraktiken implementieren.

\section{Anwendungsbeispiel: Kollaboratives Schreiben im Journalismus}

Betrachten wir ein HWA-System zur Unterstützung von Journalist*innen: Drei Journalist*innen einer überregionalen Zeitung und ein KI-basierter Assistent arbeiten als Team und nutzen ein HWA-System. Ihr gemeinsames Ziel ist das Schreiben eines Artikels zu den Auswirkungen von Homeoffice auf die Produktivität und das Wohlbefinden. Der Arbeitsprozess umfasst folgende Aufgabenbestandteile: Recherche relevanter Grundlagen, Durchführung von Interviews mit Expert*innen und Schreiben des Artikels. Um die kognitive Belastung der Journalist*innen zu reduzieren und einen qualitativ hochwertig recherchierten Artikel zu schreiben, erfolgt folgende Allokation von Aufgaben zu den jeweiligen Wissensträgern.

- Automatisierung (,AI in the Loop of Human Intelligence“): Auf Basis einer ersten, kurzen Skizze des Artikels übernimmt der KI-basierte Assistent Informationsrecherche und -verarbeitung. Hierfür extrahiert der Assistent Entitäten aus der Skizze und führt eine Suche in verschiedenen Datenbanken durch. Die Informationen werden priorisiert und dem menschlichen Verarbeitungsprozess entsprechend anschaulich aufbereitet. Sobald die Journalist*innen mit dem Schreiben des Artikels fortfahren, nutzt die KI die neuen Informationen, um die Vorschläge entsprechend anzupassen.

- Verifizierung von Wissen (,Human Intelligence in the Loop of $A I^{\prime \prime)}$ : Die Journalist*innen geben Feedback zu den von dem KI-basierten Assistenten unterbreiteten Vorschlägen, damit sich der KI-basierte Assistent bei der Informationsrecherche verbessern kann. Dazu bewerten die Journalisten die vom KI-System gelieferten Informationen, Vorschläge, wie z. B. Studien, Statistiken oder Experten hinsichtlich Relevanz und Seriosität.

- Augmentierung („AI in the Loop of Human Intelligence"): Die Journalist*innen widmen sich kreativen Aufgaben wie der Entwicklung einer „Story“, um die Aufmerksamkeit der Leser*innen zu halten oder der Durchführung von Interviews.

\section{Forschungsziele und Ausblick}

Hybride Wissensarbeit und HWA-Systeme beschreiben ein im Entstehen befindliches Forschungsgebiet soziotechnischer Arbeitssysteme, die hybride Zusammenarbeit und lebenslanges Lernen von Menschen und System gleichermaßen ermöglichen. Um HWA-Systeme zu entwickeln, ist Gestaltungswissen hinsichtlich (i) des sozialen Subsystems, insbesondere Wissen über die Anatomie der Zusammenarbeit und des Lernens von Menschen sowie Collaboration Engineering und (ii) des technischen Subsystems, insbesondere Wissen über CIL-Techniken und aktives Lernen, erforderlich.

Förderung The research leading to the presented results was conducted in the context of the project HyMeKI (funded by the Federal Ministry of Education and Research (BMBF), 01IS20057)

Funding Open Access funding enabled and organized by Projekt DEAL.

Open Access Dieser Artikel wird unter der Creative Commons Namensnennung 4.0 International Lizenz veröffentlicht, welche die Nutzung, Vervielfältigung, Bearbeitung, Verbreitung und Wiedergabe in jeglichem Medium und Format erlaubt, sofern Sie den/die ursprünglichen Autor(en) und die Quelle ordnungsgemäß nennen, einen Link zur Creative Commons Lizenz beifügen und angeben, ob Änderungen vorgenommen wurden.

Die in diesem Artikel enthaltenen Bilder und sonstiges Drittmaterial unterliegen ebenfalls der genannten Creative Commons Lizenz, sofern sich aus der Abbildungslegende nichts anderes ergibt. Sofern das betreffende Material nicht unter der genannten Creative Commons Lizenz steht und die betreffende Handlung nicht nach gesetzlichen Vorschriften erlaubt ist, ist für die oben aufgeführten Weiterverwendungen des Materials die Einwilligung des jeweiligen Rechteinhabers einzuholen.

Weitere Details zur Lizenz entnehmen Sie bitte der Lizenzinformation auf http://creativecommons.org/licenses/by/4.0/deed.de.

\section{Literatur}

1. Akata Z, Balliet D, de Rijke M et al (2020) A research agenda for hybrid intelligence: augmenting human intellect with collaborative, adaptive, responsible, and explainable artificial intelligence. Computer 53:18-28 
2. Alavi M, Leidner DE (2001) Review: knowledge management and knowledge management systems: conceptual foundations and research issues. MISQ 25:107

3. Alter S (2017) Work system theory and work system method: a bridge between business and IT views of IT-reliant systems in organizations. Proceedings of the 10th Innovations in Software Engineering Conference

4. Arlinghaus A (2017) Wissensarbeit: Aktuelle arbeitswissenschaftliche Erkenntnisse. Hans-Böckler-Stiftung, Düsseldorf

5. Dalkir K (2005) Knowledge management in theory and practice. Routledge, Amsterdam, London

6. Dellermann D, Calma A, Lipusch N, Weber T, Weigel S, Ebel P (2019) The future of human-AI collaboration: a taxonomy of design knowledge for hybrid intelligence systems. In: Bui T (Hrsg) Proceedings of the 52nd Hawaii International Conference on System Sciences: Hawaii International Conference on System Sciences

7. Greiner ME, Böhmann T, Krcmar H (2007) A strategy for knowledge management. J Knowl Manag 11:3-15

8. Haenlein M, Kaplan A (2019) A brief history of artificial intelligence: on the past, present, and future of artificial intelligence. Calif Manage Rev 61:5-14

9. Leimeister JM (2010) Collective intelligence. Bus Inf Syst Eng 2:245-248

10. Leimeister JM (2014) Collaboration engineering. Springer, Berlin, Heidelberg
11. Pyöriä P (2005) The concept of knowledge work revisited. J Knowl Manag 9:116-127

12. Salaberry MR (2018) Declarative versus procedural knowledge. In: Liontas JI, DelliCarpini M (Hrsg) The TESOL encyclopedia of English language teaching. Wiley-Blackwell, Hoboken

13. Schmitz C, Hotho A, Jäschke R, Stumme G (2006) Kollaboratives Wissensmanagement. In: Pellegrini T, Blumauer A (Hrsg) Semantic web. Springer, Berlin, Heidelberg

14. Sick B, Oeste-Reiß S, Schmidt A, Tomforde S, Zweig AK (2018) Collaborative interactive learning. Informatik Spektrum 41:52-55

15. Thordsen T, Murawski M, Martensen M, Rademacher C, Bick M (2020) Digitale Kompetenzen - Welche Anforderungen stellt die Digitalisierung an Wissensarbeiter und Unternehmen? In: Heisig et al (Hrsg) WM in digitalen Arbeitswelten - Aktuelle Ansätze und Perspektiven

16. Traumer F, Oeste-Reiß S, Leimeister JM (2017) Towards a Future Reallocation of Work between Humans and Machines-Taxonomy of Tasks and Interaction Types in the Context of Machine Learning. In: International Conference on Information Systems (ICIS). Seoul, South Korea

17. de Vreede G-J, Briggs RO (2019) A Program of Collaboration Engineering Research and Practice: Contributions, Insights, and $\mathrm{Fu}-$ ture Directions. J Manag Inf Syst 36:74-119 